\title{
Injury of Cervical Esophagus: The Importance of a Proper
}

\section{Management}

\author{
Giordano D* \\ Otolaryngology Unit, Department of Surgery, Azienda USL-IRCCS di Reggio Emilia, \\ Italy \\ *Corresponding author: Davide Giordano, MD, Otolaryngology Unit, Department of \\ Editorial \\ Volume 4 Issue 2 \\ Received Date: July 29, 2019 \\ Published Date: August 01, 2019 \\ DOI: $10.23880 /$ ooaj-16000183
}

Surgery, Azienda USL-IRCCS di Reggio Emilia, viale Risorgimento 80, 42123 Reggio Emilia, Italy, Tel: 00390522296273 ;

Fax: 00390522 295839; Email: Davide.Giordano@ausl.re.it

\section{Editorial}

The Injury of cervical esophagus is a rare event, but its relevance is indubitably high for a number of reasons. First, it has a significant morbidity and sometimes can lead the patient to death. Second, it requires a multidisciplinary approach, and then, an adequate setting for diagnosis and treatment. Third, rapid diagnosis and prompt treatment are crucial for the patient, as many experiences reported in literature underline the fact that delayed treatment is associated to an increased morbidity and mortality.

Iatrogenic (esophagoscopy, trans-esophageal echocardiography), spontaneous hemetogenic perforations, and perforations resulting from extraction of foreign bodies (i.e.: dental prosthesis) are the most common conditions in clinical practice. Instead, penetrating injuries of the neck (gunshot, stab) represent the main mechanism of traumatic lesion of the cervical esophagus. Blunt trauma rarely causes an esophageal perforation.

Diagnosis is often evident on the basis of the history. The patient may present non-specific signs and symptoms of esophageal perforation, such as hemoptysis, hematemesis, hoarseness, dysphagia, and dyspnoea. On physical exam, the main sign is represented by subcutaneous emphysema of the neck that can rapidly evolve in neck swelling. The pulsatile feature of the neck swelling should suggest a vascular injury. An efficient evaluation can lead to a rapid confirmation of the diagnosis, as well as a proper identification of perforation. This is crucial in order to plan a prompt treatment, thus reducing the risk of unfavorable course. X-ray of the chest and abdomen is the standard baseline exam that can show air in the neck, with or without mediastinal emphysema, even if these signs are not diagnostic for esophageal perforation. Esophagogram with water-soluble contrast represents an effective tool for achieving diagnosis of esophageal perforation. Some authors report that esophagoscopy followed by contrast esophagogram may lead to correct diagnosis in the vast majority of cases. The role of CT scan in confirming diagnosis and identifying the site of perforation is limited. Nevertheless, it is useful for surgical planning, as it adequately characterizes fluid collections that have to be debrided, and for postoperative follow-up, to evaluate the need of surgical re-exploration.

The importance of proper diagnosis in such cases is given by the fact that progressive spread of emphysema through the deep neck spaces, and then to the mediastinum, causes imbibition of these spaces with gastric fluids, and saliva. This situation may result in a rapid infection of the mediastinum known as "descending mediastinitis", a potentially catastrophic condition in a large part of cases.

Initial management of a stable patient with esophageal perforation consists in "nil per os" status, broad spectrum antibiotherapy, and internal drainage, when possible, by careful, preferably under endoscopic guidance, positioning of a nasogastic tube. For unstable patients, surgical exploration is mandatory, as it is directed to lifethreatening conditions. Surgical management of perforation of the cervical esophagus is performed by a trancervical prevascular approach, with an incision along 


\section{Otolaryngology Open Access Journal}

the anterior border of the sternocleidomastoid muscle, section of the omo-hyoid muscle and the middle thyroid vein. Then, the esophagus is exposed along its cervical extension behind the trachea, and medially to the great vessels of the neck. Repair of the injury may be made by direct closure, layer by layer. Sometimes, when collection is present, surgical repair can require debridement of necrotic tissues. In both cases a pedicled flap of sternocleidomastoid muscle, or homo-hyoid muscle can be used to reinforce the suture. Rarely, in case of wide perforations, direct suture cannot be feasible, and a gastric pull-up could be necessary.

Once again, the timing of surgery is crucial. In fact, delayed surgical exploration can facilitate infection. In such case, necrotic tissues, and fluids collections makes it difficult to identify of perforation or repair of the injury.

In conclusion, injury of cervical esophagus, although rare, represents a relevant event in urgent setting. Given the morbidity and mortality of this picture, hospital setting has to be ready to provide a rapid and efficient response in terms of diagnosis. Treatment should be addressed to perforation repair and to other concurrent injuries in order to preserve vital functions and avoid infective complications (Figure 1).

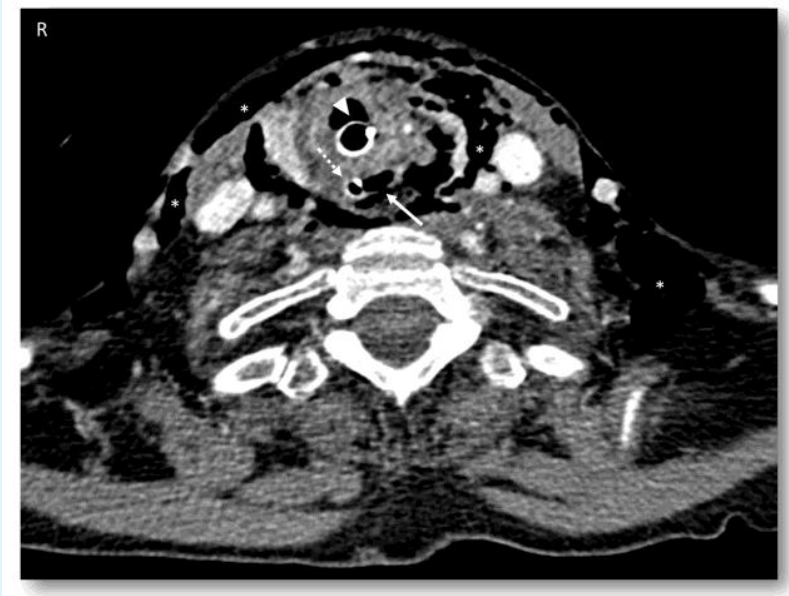

Figure 1: Contrast-enhanced CT scan showing a wide emphysema (asterisks) due to iatrogenic esophageal perforation (arrow). The patient underwent earlier orotracheal intubation (arrowhead) and nasogastic tube insertion (dotted arrow). Exam performed seven hours after injury. 